\title{
Atypical Case of Partial Anomalous Pulmonary Venous Connection Misdiagnosed as Primary Pulmonary Arterial Hypertension
}

\author{
Shuyang Lu, ${ }^{1,2^{*}}$ Lai Wei, ${ }^{1,2^{*}}$ Jiayu Zheng, ${ }^{1,2}$ Chunsheng Wang ${ }^{1,2}$ \\ ${ }^{1}$ Shanghai Institute of Cardiovascular Disease, China; ${ }^{2}$ Department of Cardiovascular Surgery, Zhongshan Hospital, Fudan \\ University, Shanghai, China
}

\section{ABSTRACT}

In this case report, we present the delayed diagnosis of an atypical partial anomalous pulmonary venous connection, which was initially misdiagnosed as primary pulmonary arterial hypertension. It was difficult to make a conclusive diagnosis using transthoracic echocardiography (TTE), because the two high right superior pulmonary veins drained into the superior vena cava and limited the shunt of patent foramen ovale. Preoperative TTE only showed right heart volume overload, pulmonary arterial hypertension, and severe tricuspid valve insufficiency. A chest CT-angiography (angio-CT) finally found the anomalous right superior pulmonary venous connection, which was further confirmed by surgery. The postoperative course was uneventful.

\section{INTRODUCTION}

A partial anomalous pulmonary venous connection (PAPVC) is characterized by the failure of one or more of the pulmonary veins to drain into the left atrium, causing a left-to-right shunt. This congenital disease is frequently associated with atrial septal defects (ASD). Both PAPVC and ASD can cause pulmonary arterial hypertension (PAH), right heart volume overload, and tricuspid valve insufficiency [Sahay 2012]. TTE is sensitive to detect the secondary signs and ASD. However, PAPVC is often overlooked because of the extracardiac drainage. Our primary aim was to present the delayed diagnosis of PAPVC and ASD, and discuss the role of angio-CT in the diagnosis of this rare congenital anomaly. The secondary aim was to share our surgical experiences.

Received fune 3, 2016; received in revised form December 8, 2016; accepted fanuary 13, 2017.

*Both authors contributed equally to this work.

This work is supported by the National Natural Science Foundation of China (grant 81501595) and the foint Research of Major Diseases, Shanghai (grant 2014ZYFB0402).

Correspondence: Chunsheng Wang, MD, PhD, Fenglin Road 180, Xubui District, Shanghai 200032, China; +8621-64041990-2504; fax: +8621-640419902645 (e-mail: wang_cs@126.com).

\section{CASE REPORT}

A 27-year-old female was referred for further treatment, having a previous diagnosis of severe primary PAH (pulmonary artery systolic pressure [PAP] $110 \mathrm{mmHg}$ ). Her past medical history included two abortions. At the second time, TTE results indicated an enlargement of the right heart, mild tricuspid valve insufficiency, and severe PAP. No further abnormalities were found and the patient was diagnosed as having primary PAP.

In the past three months, the patient presented with progressive shortness of breath, palpitations, and fatigue. TTE examination at our hospital was in accordance with her former results. Neither ASD nor PAPVC was conclusive. Right heart catheterization revealed that the venous oxygen saturation of superior vena cava, right atrium, inferior vena cava, right ventricle, and pulmonary artery were $91 \%, 86 \%$, $82 \%, 87 \%$, and $88 \%$, respectively; PAP, $113 \mathrm{mmHg}$; and the pulmonary vascular resistance, $518.2 \mathrm{dyn} / \mathrm{s.cm} .5$ multi-slice angio-CT showed two high right superior pulmonary veins draining towards the superior vena cava (Figure 1).

Under general anesthesia and endotracheal intubation, the patient underwent surgical treatment by redirection of anomalous right superior pulmonary veins into the left atrium. The right superior vena cava (SVC) was circumferentially dissected out from the SVC-right atrial junction at the level of the innominate vein. The azygos vein was reserved.
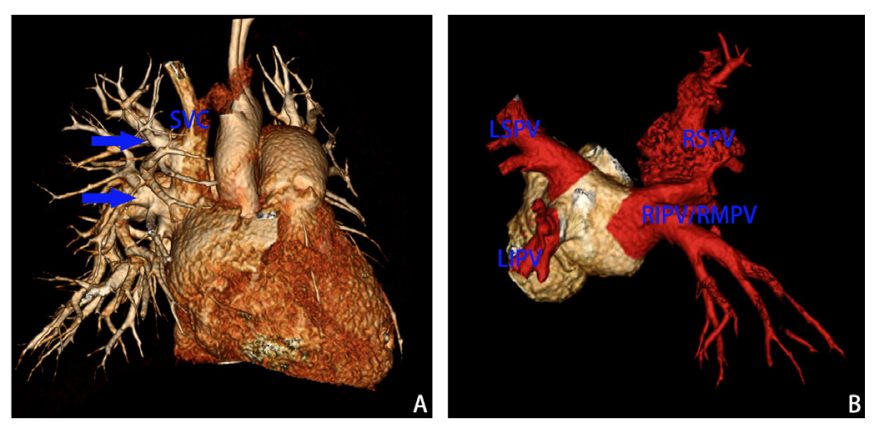

Figure 1. Preoperative CTV. (A) Multi-slice angio-CT showed two high RSPVs (blue arrows) draining towards the superior vena cava (anterior view). (B) RSPV did not connect with LA (posterior view). RSPV indicates right superior pulmonary vein; LA, left atrium; SVC, superior vena cava; RIPV/RMPV, right inferior/middle pulmonary veins; LSPV, left superior pulmonary vein; LIPV, left inferior pulmonary vein. 

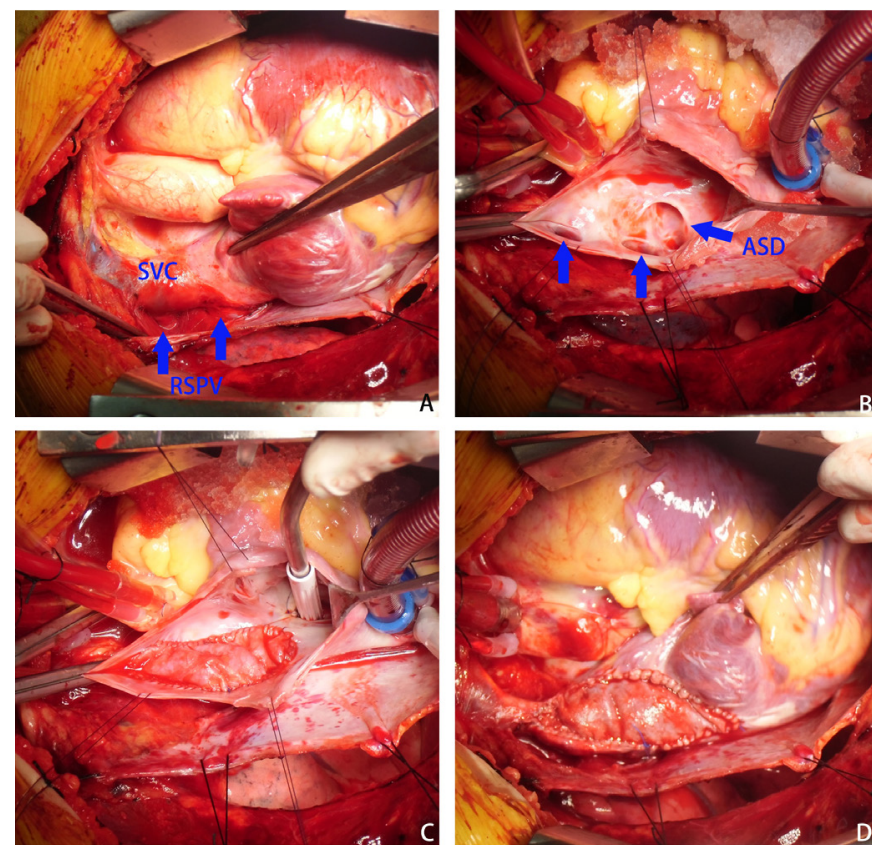

Figure 2. Surgical treatment with one patch method. (A, B) Two RSPVs were confirmed draining into the superior vena cava and the overlooked ASD was found (blue arrows). (C, D) PAPVC was successfully treated with an autologous pericardial patch. RSPV indicates right superior pulmonary vein; SVC, superior vena cava; PAPVC, partial anomalous pulmonary venous connection; ASD, atrial septal defect.

Cardiopulmonary bypass was established with cannulation of the ascending aorta, jugular vein, and inferior vena cava (IVC). Two right superior pulmonary veins were confirmed draining into the superior vena cava (Figure 2, A). After achieving mild hypothermia, the ascending aorta was cross clamped, cold-blood cardioplegia administered, and cardiac arrest achieved. Combined incisions of the right atrium and SVC were made, and the two orifices of the anomalous pulmonary veins and the overlooked ASD were found (Figure 2, B). An autologous pericardial patch treated with $0.6 \%$ gluteraldehyde for $3 \mathrm{~min}$ was sutured to the margin of ASD onto the superior margin of PAPVC (Figure 2, C). ASD was enlarged to create a non-restrictive flow of the pulmonary veins to the left atrium. Right atrium and SVC incisions were also closed with autologous pericardial patches to avoid stenosis of SVC (Figure 2, D). The patient was gradually weaned off bypass and the ventilator support removed during the first 12 hours. All vital signs were stable. Postoperative angio-CT showed that PAPVC was successfully corrected (Figure 3 ). No complications were observed during the perioperative period. At the 14-month follow-up, echocardiography showed that the pulmonary pressure was reduced to $38 \mathrm{mmHg}$, and the patient returned to normal activity. At the time of writing of this report, the subject remains asymptomatic.

\section{DIscussioN}

We present a rare case of atypical anatomical variation of PAPVC, which was overlooked in the beginning. The initial
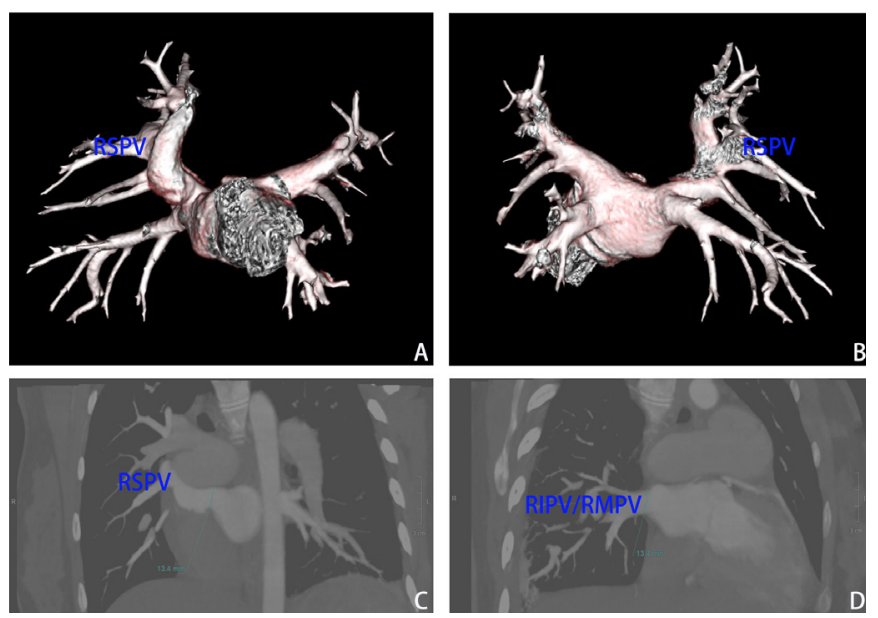

Figure 3. Postoperative CTV. (A, B) Anterior and posterior views show RSPVs draining into LA. (C, D) Postoperative diameter of RSPV was comparable with RIPV. RSPV indicates right superior pulmonary vein; LA, left atrium; RIPV/RMPV, right inferior/middle pulmonary veins.

diagnosis of most PAPVC is often made by TTE [Szychta 2015]. However, this case was delayed due to misdiagnosis by TTE both at our hospital and at a local hospital. TTE found only structural and hemodynamic changes, such as right heart volume overload, tricuspid valve insufficiency, and PAH. The probable reasons for misdiagnosis included doctors' experiences and special anatomical variation. Angio-CT is a reliable tool for the diagnosis of PAPVC with unexplained pulmonary hypertension, and has gradually replaced cardiac catheterization, especially when venous anatomy is complex or when TTE is suboptimal [Wang 2014]. Amat et al reported that angio-CT had 100\% sensitivity for diagnosing PAPVC in patients with SV-ASD, $100 \%$ specificity, $100 \%$ positive predictive value, and $100 \%$ negative predictive valve [Amat 2011]. Owing to the increased use of CT to image coronary arteries in adults, more frequent diagnosis of PAPVC is expected in future.

Surgery is the definitive treatment for PAPVC. However, it is a challenge for clinicians to determine the true hemodynamic impact of PAPVC in order to prevent unnecessary surgery, while not withholding therapy in patients who would really benefit from it. Indications for surgical treatment include significant left-to-right shunt (at least 2:1), compression or obstruction of the surrounding structures caused by the anomalous vein, recurrent pulmonary infections, and concomitant surgical repair of other major cardiac lesions [Glaveckaite 2014]. We thought that our patient had significant shunting because she was symptomatic and had right heart dilatation, volume overload, and severe pulmonary arterial hypertension. Therefore, she was a proper candidate for surgery.

Several techniques have been described in the repair of a PAPVC to the SVC. In the present case, we used one-patch diversion procedure; the right atrium incision was extended into the SVC, and an autologous pericardial patch was placed to create a pulmonary vein return route [Seike 2011]. Some authors think that an incision or suturing through the anterior 
right atrial-SVC junction may cause SVC stenosis, pulmonary vein stenosis, and sinoatrial node dysfunction [Kottayil 2011]. Therefore, Warden and colleagues reported a technique in which the SVC was divided, the cephalic SVC was anastomosed to the right atrial appendage, and the caudal SVC served as a conduit for pulmonary venous drainage to the left atrium [Warden 1984]. However, we think that the Warden procedure cannot eliminate the risk of sinus dysfunction. Furthermore, when anomalous pulmonary veins connect to high SVCs, the cephalic end of the divided SVC becomes more distant from the right atrial appendage, and it is easy to develop tension and later stenosis of cavoatrial anastomosis. We did not adopt this method because of these reasons. In our opinion, there is not a perfect technique tailored to encompass all patients. The surgical experience of the physician is also very important. The postoperative course of our patient was uneventful.

In conclusion, this case reminds us to consider a broad differential when faced with unexplained pulmonary arterial hypertension. Angio-CT is an effective tool for the diagnosis of PAPVC and surgery is the definitive treatment for a patient with significant left-to-right shunt due to PAPVC.

\section{REFERENCES}

Amat F, Le Bret E, Sigal-Cinqualbre A, et al. 2011. Diagnostic accuracy of multidetector spiral computed tomography for preoperative assessment of sinus venous atrial septal defects in children. Interact Cardiovasc Thorac Surg 12:179-82.

Glaveckaite S, Lusaite K, Grabauskiene V, Valeviciene N, Laucevicius A. 2014. Delayed diagnosis of an isolated partial anomalous pulmonary venous connection. Cent Eur J Med 9:508-12.

Kottayil BP, Dharan BS, Menon S, et al. 2011. Anomalous pulmonary venous connection to superior vena cava: Warden technique. Eur J Cardiothorac Surg 39:388-91.

Sahay S, Krasuski RA, Tonelli AR. 2012. Partial anomalous pulmonary venous connection and pulmonary arterial hypertension. Respirology 17:957-63.

Seike Y, Nakamura Y, Tagusari O, Domoto S, Nakano K, Nagashima M. 2011. Partial anomalous pulmonary venous connection and partialatrioventricular septal defect in an adult: report of a case. Surg Today $41: 1421-3$

Szychta W, Cerin G, Popa BA, et al. 2015. Atypical abnormal pulmonary vein drainage with atrial septal defect: surgical treatment. Echocardiography 32:1040-3.

Wang H, Guan H, Wang DW. 2014. Partial anomalous pulmonary venous connection to superior vena cava that overrides across the intact atrial septum and has bi-atrial connectionin a 75-year-old female presenting with pulmonary hypertension. BMC Cardiovasc Disord 14:149.

Warden HE, Gustafson RA, Tarnay TJ, Neal WA. 1984. An alternative method for repair of partial anomalous pulmonary venousconnection to the superior vena cava. Ann Thorac Surg 38:601-5. 